\title{
Crystallization and Characterization of a New Nonlinear Optical Crystal: L-Proline Succinate (LPS)
}

\author{
P. Paramasivam ${ }^{1}$, C. Ramachandra Raja ${ }^{2 *}$ \\ ${ }^{1}$ Anjalai Ammal Mahalingam Engineering College, Kovilvenni, India; ${ }^{2}$ Department of Physics, Govt Arts College (Autonomous), \\ Kumbakonam, India. \\ Email: "crraja_phy@yahoo.com
}

Received November $7^{\text {th }}, 2011$; revised December $9^{\text {th }}$, 2011; accepted December $18^{\text {th }}, 2011$

\begin{abstract}
In this analysis, the single crystal of L-Proline Succinate (LPS) has been successfully synthesized and the purity of material has been increased by repeated recrystallization process. Single crystal was grown by adopting the method of growing in a slow evaporation solution using water as solvent at room temperature. The LPS single crystal has been synthesized by taking equimolar quantity of L-Proline and succinic acid, by mixing them thoroughly using deionized water. The prepared concentrated solution was placed in an undisturbed condition, and the solution was inspected regularly. The single crystal has been harvested over a period of 1 month. The same crystal was characterized by different techniques for finding its suitability for device fabrications. The grown crystal was characterized by Single crystal XRD, Powder XRD, FTIR, UV-vis-NIR, DTA/TGA and SHG analyses, respectively. The observed results from various characterization show the suitability for NLO application. The second harmonic generation of this grown crystal was checked using Kurtz Perry technique which showed positive results. The UV cut-off wavelength and the decomposition temperature of this grown crystal were found to be good when compared with the existing organic crystals.
\end{abstract}

Keywords: Slow Evaporation; Crystal Growth; X-Ray Diffraction; Fourier Transform Infrared Spectroscopy; Second Harmonic Generation; LPS Crystal

\section{Introduction}

One of the most important applications of NLO materials is their use for fast data transfer, combined with a very high Signal-to-Noise ratio, even over long distances. In recent years, different applications of NLO and photorefractive materials have been developed, for example, optical frequency conversion, electro-optical modulation, dynamic holography, optical writing and optical guiding of laser beams [1]. It is seen that L-proline and ( $4 R$ )-hydroxy-Lproline derivatives, containing donor groups are chiral carriers [2]. The introduction of chirality by means of an asymmetrically substituted carbon should in addition respect the molecular features leading to a high nonlinear behaviour [3]. Proline and its derivatives are often used as asymmetric catalysts in organic reactions.

Only noncentrosymmetric alignment of the chromophores in the crystal lattice leads to an observable bulk secondorder NLO response [4]. In order to obtain the adjustment of the nonlinear efficiency/transparency, based on the molecular engineering and crystal engineering approach, it is tried to develop a new method to design organic nonlinear optical second-harmonic generation materials such

${ }^{*}$ Corresponding author. as organic inclusion complex [5]. In this study, the crystal growth of a new NLO crystal of L-Proline succinate by slow evaporation technique and its characterization along with its optical properties is reported.

\section{Experimental Details}

Equal proportions of L-Proline and succinic acid were taken and were dissolved separately in deioinized water. Then the solution of L-Proline was poured into the dissolved succinic acid mixture. The solution thus arrived was filtered twice to remove dust particles and undissolved materials. The reaction takes place between L-Proline and succinic acid (acid-based) through hydrogen transfer. Thus formed ionic compound of L-Proline succinate is represented in the following equation:<smiles>O=C(O)C1CCCN1</smiles><smiles>CC(CC(=O)O)CC(=O)OC(=O)CCCC(=O)O</smiles>

The saturated solution was maintained in the undisturbed condition and the beaker was covered by polythene paper. Few holes were made on the polythene cover for slow evaporation. By adopting the solution growth method, 
single crystal of L-Proline succinate (LPS) was grown from supersaturated solution at room temperature. Then this solution was periodically inspected and from the $20^{\text {th }}$ day onwards the crystal started growing and it was permitted to grow for another 10 days in order to get a nominal size suitable for characterization. The single crystal of LPS with dimensions of $8 \mathrm{~mm} \times 5 \mathrm{~mm} \times 10 \mathrm{~mm}$ was thus obtained. The L-Proline interacts with succinic acid through a single N-H-O hydrogen bond. A single crystal of LPS which has been grown by this process is shown in Figure 1.

\section{Characterization}

The lattice parameters and the crystal systems have been determined using single crystal X-ray diffraction analysis (Model: Bruker AXS Kappa APEX II single crystal CCD diffractometer). The functional groups presented in the LPS compound have been identified by Bruker IFS 66V model FTIR Spectrometer using $\mathrm{KBr}$ pellet technique in the region $400-4000 \mathrm{~cm}^{-1}$. Optical behaviour of LPS was measured by Perkin Elmer Lambda 35 UV-VIS-NIR Spectrophotometer in the wavelength range of $190-1100 \mathrm{~nm}$. The thermal stability of LPS was studied by thermo gravimetric analysis (TGA) and differential thermal analysis (DTA) by using SDT Q600 V8.3 Build 101 thermal analyzer instrument ranging from room temperature to $1100^{\circ} \mathrm{C}$ at a heating rate of $20^{\circ} \mathrm{C}$ per minute under nitrogen atmosphere.

\section{Results and Discussion}

\subsection{Single Crystal X-Ray Diffraction Analysis}

Single crystal X-ray diffraction studies were carried out on the grown crystals. The X-ray data were collected using X-ray diffractometer (Model: Bruker AXS Kappa APEX II single crystal CCD). The observed results indicate that the crystal belongs to monoclinic crystal system and the determined unit cell parameters are $\mathrm{a}=5.07 \AA, \mathrm{b}=8.84$ $\AA, \mathrm{C}=5.48 \AA, \alpha=90^{\circ}, \beta=91.60^{\circ}, \gamma=90^{\circ}$ and $\mathrm{V}=246 \AA^{3}$.

\subsection{FTIR Spectroscopy}

The functional groups presented in the LPS compound have been identified by Bruker IFS 66V model FTIR Spectrometer using $\mathrm{KBr}$ pellet technique in the region 400 $4000 \mathrm{~cm}^{-1}$. The FTIR spectrum of title compound is shown in Figure 2. The peaks obtained are $3419 \mathrm{~cm}^{-1}$, due to stretching vibration of $\mathrm{CH}$ and the peak at $1600 \mathrm{~cm}^{-1}$ is due to the stretching vibration of $\mathrm{C}=\mathrm{O}$. The bands appeared at $793 \mathrm{~cm}^{-1}$ is assigned unambiguously to the wagging of $\mathrm{NH}_{2}$ modes. The $\mathrm{OH}$ stretching vibrations is assigned in the range of $2565 \mathrm{~cm}^{-1}$. The peak at $1398 \mathrm{~cm}^{-1}$ is due to the symmetric stretching of $\mathrm{COO}^{-}$. These assignments are also supported in the literature [6-10]. The observed bands along with their vibrational assignments are given in Table $\mathbf{1 .}$

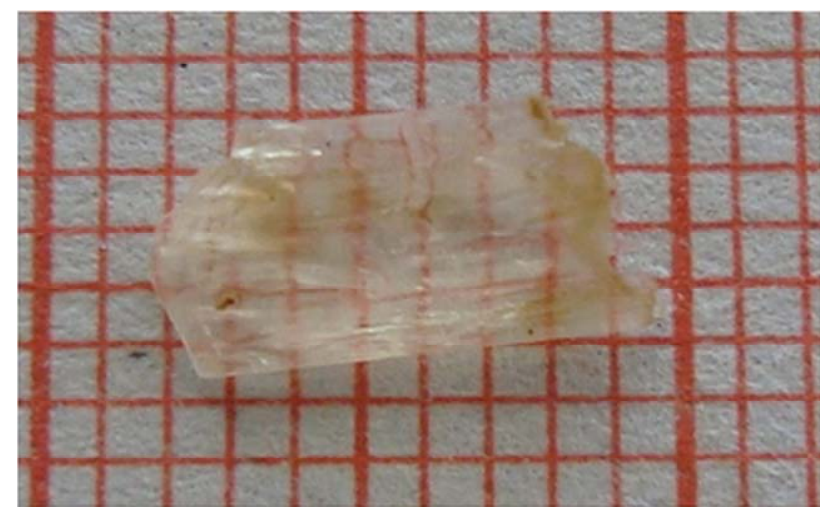

Figure 1. Photograph of LPS crystal.

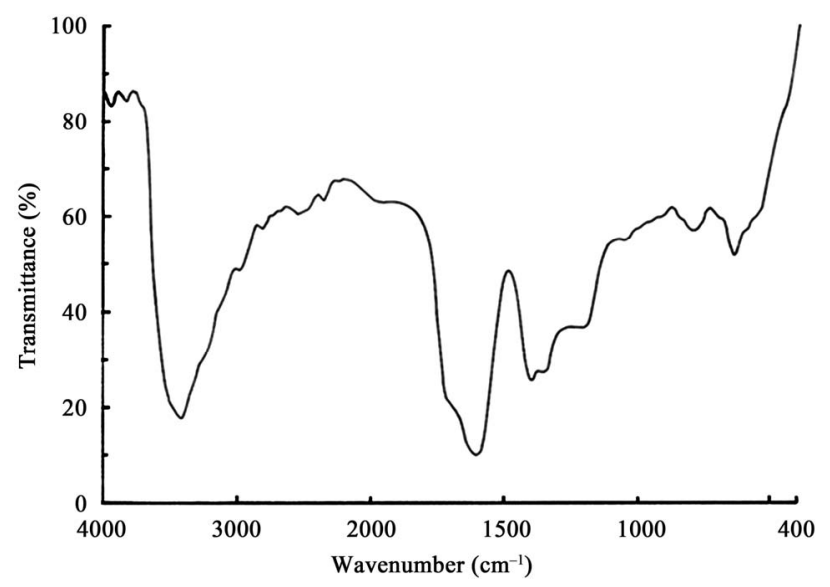

Figure 2. FTIR spectrum of LPS crystal.

Table 1. The observed frequencies and corresponding assignments.

\begin{tabular}{cl}
\hline Observed FTIR frequencies & Assignments \\
\hline 3419 & $\mathrm{CH}$ stretching \\
1600 & $\mathrm{C}=\mathrm{O}$ stretching \\
1398 & Symmetric stretching of $\mathrm{COO}^{-}$ \\
793 & $\mathrm{NH}_{2}$ wagging \\
2565 & $\mathrm{O}-\mathrm{H}$ stretching \\
\hline
\end{tabular}

\subsection{UV-Visible Spectroscopy}

Good optical transmittance and lower cut-off wavelengths are very important properties for NLO crystals. Optical behaviour of LPS was measured by Perkin Elmer Lambda 35 UV-VIS-NIR spectrophotometer in the wavelength range of $190-1100 \mathrm{~nm}$. The recorded spectrum is shown in Figure 3. The crystals are broadly transparent possessing a transmission of greater than $90 \%$ for light with incident wavelengths from 236 - $1100 \mathrm{~nm}$. The UV transparency cut-off wavelength of LPS crystal occurs at 204 $\mathrm{nm}$ which is better than L-Prolinium tartrate and 4phenylpridinium hydrogen squarate [11,12]. It is observed 


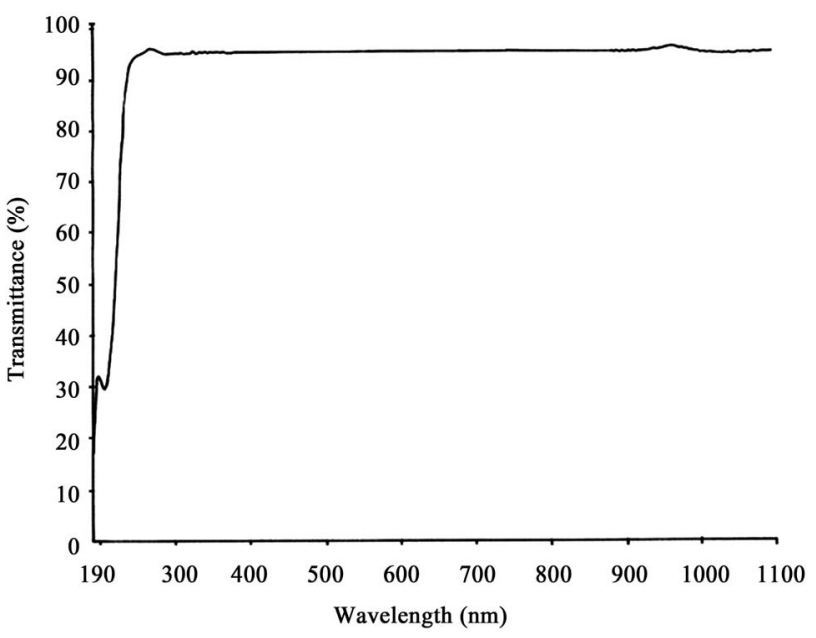

Figure 3. Transmission spectrum of LPS crystal.

that in the LPS crystal, there is high transmittance in the far ultraviolet, visible and infra red region. Hence, the title compound may be used for the nonlinear optical applications in the above mentioned wavelength range.

\subsection{Second Harmonic Generation}

The SHG of the crystal was checked using the powder SHG technique developed by Kurtz and Perry [13]. A Qswitched Nd:YAG laser beam of wavelength $1064 \mathrm{~nm}$, with beam energy of $4.5 \mathrm{~mJ} /$ pulse, and pulse width of $8 \mathrm{~ns}$ with a repetition rate of $10 \mathrm{~Hz}$ were used. The grown single crystal was crushed to fine powder and then packed in a micro capillary of uniform bore and exposed to laser radiations. The $532 \mathrm{~nm}$ radiation was collected by a monochromater after separating the $1064 \mathrm{~nm}$ pump beam with an infra-red blocking filter. The second harmonic radiation generated by the randomly oriented micro crystals was focused by a lens and detected by a photo multiplier tube (Hamamatsu R2059). The second harmonic generation is confirmed by the emission of green light and its efficiency is found to be $23 \%$ of that of KDP crystal.

\subsection{Thermal Analysis}

The thermal behaviour of LPS had been studied by thermo gravimetric analysis (TGA) and differential thermal analysis (DTA) using SDT Q600 V8.3 Build 101 thermal analyzer instrument, ranging from room temperature to $1100^{\circ} \mathrm{C}$ at a heating rate of $20^{\circ} \mathrm{C}$ per minute under nitrogen atmosphere. TGA-DTA curve of L-Proline succinate is shown in Figure 4. From the TGA curve, the material starts to decompose around $160^{\circ} \mathrm{C}$, which is also confirmed by the peak which appears at $154^{\circ} \mathrm{C}$ in the DTA curve. The thermal stability of LPS is low compared to L-Proline $\left(205^{\circ} \mathrm{C}\right)$ and succinic acid $\left(240^{\circ} \mathrm{C}\right)$. The TGA curve shows a major loss of weight and two losses of smaller weight. The major weight loss of $86.58 \%$ is observed between

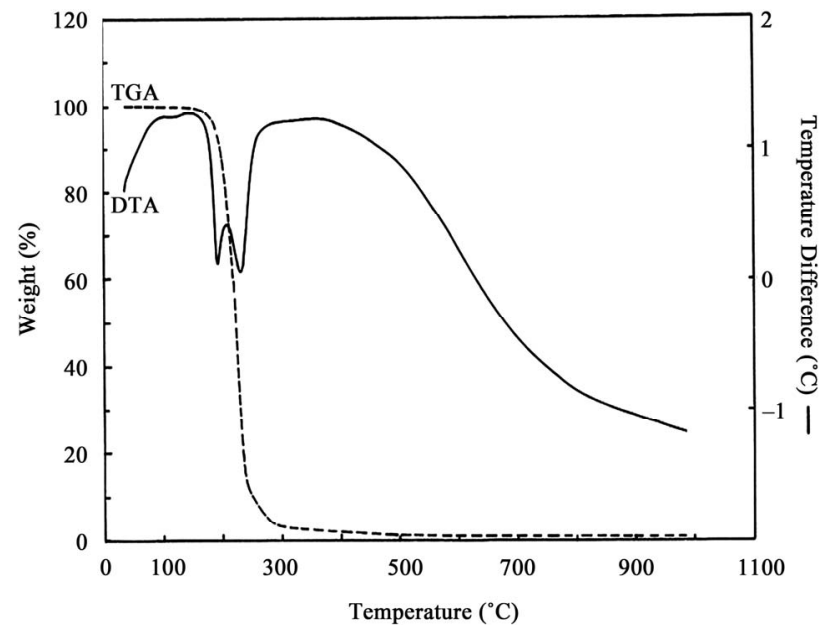

Figure 4. TGA/DTA curve of LPS crystal.

$169.91^{\circ} \mathrm{C}$ and $243.16^{\circ} \mathrm{C}$. The decomposition of L-Proline succinate (87.53\%) leads to the major loss of weight in the above mentioned temperature region. Further, a small loss of weight of $10.24 \%$ is observed, which may be due to the decomposition of $\mathrm{C}_{2} \mathrm{H}_{2}$ (11.16\%). During the next stage of decomposition, fraction amount of hydrogen may be decomposed. A residue of $0.9018 \%$ which may be due to presence of some fraction of carbon molecule is observed. The thermal stability of the LPS single crystal is more than glycine nitrate [14] and is lower than gamma glycine and glycine acetamide $[13,15]$.

\section{Conclusion}

We have synthesized a new non-linear optical crystal with an interesting hydrogen bonding network that holds together the L-Proline and succinic acid molecules. The grown crystals are characterized by different instrumental techniques. The single crystal XRD studies prove that the grown LPS crystals belong to monoclinic crystal system. The particle size of the grown crystal is characterized by Powder XRD analysis. The presence of the functional groups of the grown crystal has been confirmed by FTIR analysis. From the optical transmittance spectrum, it is observed that there is high transmittance in the far ultraviolet, visible and near infra red regions. The UV transparency cut-off wavelength of LPS crystal occurs at 204 nm. The Kurtz Perry technique for second harmonic generation has showed positive results. It is well known that the DTA and TGA studies reveal that the crystal is thermally stable up to $160^{\circ} \mathrm{C}$.

\section{Acknowledgements}

The authors are thankful to Prof. P.K. Das, IISc, Bangalore, India for the SHG test. They also express their gratitude to the authorities of SAIF, IIT, Chennai, India and CECRI, Karaikudi, India for providing spectral facilities, 
to undertake this study.

\section{REFERENCES}

[1] T. M. Kolev, D. Y. Yancheva and S. I. Stoyanov, “Synthesis and Spectral Elucidation of Some Pyridinium Betaines of Squaric Acid: Potential Materials for Nonlinear Optical Applications,” Advanced Functional Materials, Vol. 14, No. 8, 2004, pp. 799-805.

[2] B. Gutierrez, N. Rubio and C. Minguillon, "Evaluation of L-Proline Derivatives as Chiral Carriers in the Separation of Enantiomers by Membrane Techniques," Journal of Desalination, Vol. 200, No. 1-3, 2006, pp. 117-119.

[3] R. Hierle, J. Badan and J. Zyss, "Growth and Characterization of a New Material for Nonlinear Optics: Methy l-3-Nitro-4-Pyridine-1-Oxide (POM)," Journal of Crystal growth, Vol. 69, No. 2-3, 1984, pp. 545-554. doi:10.1016/0022-0248(84)90366-X

[4] T. kolev, B. Stamboliyska and D. Yancheva, "Spectral and Structural Study of Two Acceptor-Substituted Pyridinium-Betaines of Squaric Acid: Promising Chromophores for Nonlinear Optical Applications," Chemical Physics, Vol. 324, No. 2-3, 2006, pp. 489-496. doi:10.1016/j.chemphys.2005.11.014

[5] F. Q. Meng, M. K. Lu, J. Chen, S. J. Zhang and H. Zeng, "Characterization of Linear and Nonlinear Optical Properties of a New Single Crystal,” Solid State Communications, Vol. 101, No. 12, 1997, pp. 925-928. doi:10.1016/S0038-1098(96)00702-8

[6] K. V. Krishna and M. Arivazhagan, "Vibrational and Normal Coordinate Analysis of Xanthine and Hypoxanthine," Indian Journal of Pure \& Applied Physics, Vol. 42, No. 12, 2004, pp. 411-418.

[7] G. Socrates, "Infrared and Raman Characteristic Group Frequencies,” 3rd Edition, Wiley, Chichester, 2001.
[8] G. Varaasyi, "Assignments for Vibrational Spectra of Seven Hundred Benzene Derivatives,” Wiley, New York, 1974.

[9] S. Gunasekaran and D. Uthra, "Fourier Transform Infrared and Fourier Transform Raman Spectra and Normal Coordinate Analysis of Ethyleneimine," Indian Journal of Pure \& Applied Physics, Vol. 46, No. 2, 2008, pp. 100105.

[10] S. Krishnan, C. J. Raj, R. Robert, A. Ramanand and S. J. Das, "Growth and Characterization of Succinic Acid Single Crystals,” Crystal Research Technology, Vol. 42, No. 11, 2007, pp. 1087-1090. doi:10.1002/crat.200710981

[11] S. A. M. Britto Dhas and S. Natarajan, "Growth and Characterization of L-Prolinium Tartrate-A New Organic NLO Material," Crystal Research Technology, Vol. 42, No. 5, 2007, pp. 471-476. doi:10.1002/crat.200610850

[12] C. R. Raja, P. Paramasivam and N. Vijayan, "Synthesis, Growth and Characterization of a New Nonlinear Optical Material: 4-Phenylpyridinium Hydrogen Squarate (4PHS)," Spectrochimica Acta Part A, Vol. 69, No. 4, 2008, pp. 1146-1149. doi:10.1016/j.saa.2007.06.014

[13] V. Krishnakumar, L. G. Prasad, R. Nagalakshmi and P. Muthusamy, "Physicochemical Properties of Organic Nonlinear Optical Crystal for Frequency Doubling: Glycine Acetamide,” Materials Letters, Vol. 63, No. 15, 2009, pp. 1255-1257. doi:10.1016/j.matlet.2009.02.052

[14] S. A. M. Britto Dhas and S. Natarajan, "Growth and Characterization of a New Organic NLO Material: Glycine Nitrate,” Optics Communications, Vol. 278, No. 2, 2007, pp. 434-438. doi:10.1016/j.optcom.2007.06.052

[15] T. Balakrishnan, R. R. Babu and K. Ramamurthi, "Growth, Structural, Optical and Thermal Properties of $\gamma$-Glycine Crystal," Spectrochimica Acta Part A, Vol. 69, No. 4, 2008, pp. 1114-1118. doi:10.1016/j.saa.2007.06.025 